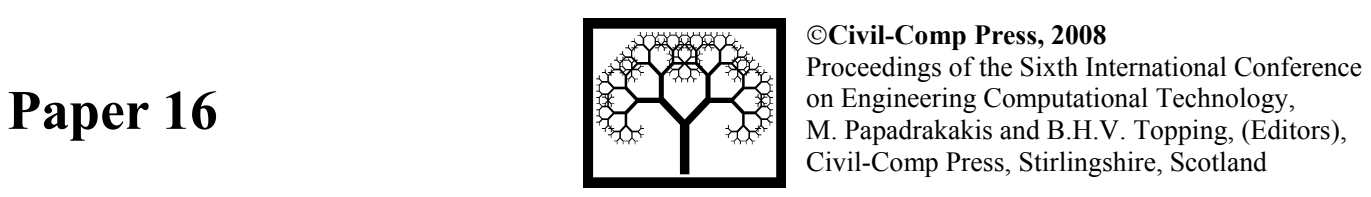

\title{
Unsteady Adaptive Stochastic Finite Elements for Quantification of Uncertainty in Time-Dependent Simulations
}

\author{
J.A.S. Witteveen and $\mathrm{H}$. Bijl \\ Faculty of Aerospace Engineering \\ Delft University of Technology, The Netherlands
}

\begin{abstract}
Due to recent advances in the development of efficient uncertainty quantification methods, the propagation of physical randomness in practical applications has become feasible for smooth and steady computational problems. The current challenges in modeling physical variability include problems with unsteadiness and discontinuous solutions. In this paper two efficient non-intrusive approaches for unsteady problems are developed based on time-independent parametrization and interpolation at constant phase. The interpolation of the samples is performed using both a global polynomial interpolation and a robust Adaptive Stochastic Finite Elements formulation with Newton-Cotes quadrature in simplex elements. Applications to an elastically mounted cylinder, a transonic airfoil flow, and an elastically mounted airfoil illustrate the efficiency, robustness, and straightforward implementation of the methodologies.
\end{abstract}

Keywords: uncertainty quantification, stochastic finite elements, fluid dynamics, fluidstructure interaction, unsteady problems, shock waves, asymptotic behavior, limit cycle oscillations, random parameters.

\section{Introduction}

Since the invention of the first modern computers in the mid-20th century, computational resources have increased by many orders of magnitude due to advances in processor clock rate and memory storage. At the same time, the efficiency of numerical algorithms has improved by even a larger factor. Numerical errors in industrial simulations, therefore, start to reach acceptable engineering levels. Nowadays, physical variability tends to dominate the error in numerical predictions. Inherent physical variations are caused by for example varying atmospheric conditions, and produc- 
tion tolerances through material properties, mass and stiffness distributions, and the geometry. Accounting for physical variations is, therefore, vital for making reliable predictions, which can be used for robust design optimization and reducing safety factors.

A straightforward uncertainty quantification method for modeling physical variations by random parameters is Monte Carlo simulation [1], in which many deterministic problems are solved for randomly varying parameter values. However, for problems which are already computationally intensive in the deterministic case, such as computational fluid dynamics and fluid-structure interaction simulations, the Monte Carlo approach can easily lead to impractically high computational costs. More efficient (non-intrusive) Polynomial Chaos methods [2, 3, 4, 5, 6, 7] have, therefore, been developed, in which the number of deterministic solves is reduced by employing a global polynomial interpolation of the samples in parameter space. An alternative sampling in suitable Gauss quadrature points is employed in Probabilistic Collocation (PC) approaches $[8,9,10]$. Due to these developments the efficient propagation of physical randomness has become possible for smooth and steady computational problems over the last decade.

The current challenges in modeling physical variability include problems with unsteadiness and discontinuous solutions. Uncertainty quantification methods usually require a fast increasing number of samples with time to resolve the large effect of random parameters in dynamical systems with a constant accuracy. It is, however, recognized in the engineering community that there is an increasing need to move towards unsteady simulations in computational fluid dynamics. This trend also dictates an increasing application of uncertainty quantification methods to time-dependent problems. Since the asymptotic effect of physical variations is of interest in, for example, post-flutter analysis of fluid-structure interaction systems [11], the increasing sample size in time can lead to thousands of required samples. The increasing number of samples with time is caused by the increasing nonlinearity of the response surface [12]. This effect is especially profound in oscillatory responses of which the frequency is affected by the random parameters. The frequency differences between the realizations lead to increasing phase differences with time, which in turn result in an increasingly oscillatory response surface and more required samples. Discontinuous responses are encountered in fluid dynamics and fluid-structure interaction in the form of, for example, shock waves in supersonic flow and bifurcation phenomena of dynamical systems. Resolving these singularities is important, since they can lead to a high sensitivity to input variation, and oscillatory or unphysical predictions by global polynomial approximations.

There is, therefore, a need to develop an efficient and robust methodology for resolving the effect of physical variations in unsteady problems with singularities. For oscillatory time-dependent responses a Fourier Chaos basis [13] has recently been proposed. Multi-element Adaptive Stochastic Finite Elements (ASFE) methods $[14,15,16,17,18]$ have been developed in order to approximate discontinuities by a more robust piecewise polynomial interpolation of the samples. 
In this paper, a combined methodology for unsteady problems with discontinuities is developed. Two efficient approaches for unsteady oscillatory problems are proposed, which both achieve a constant accuracy in time with a constant number of samples, in contrast with the usually fast increasing number of samples for other methods. The two approaches are based on:

1. Application of the uncertainty quantification interpolation to a time-independent parametrization of the oscillatory samples instead of to the time-dependent samples themselves, which results in a time-independent interpolation error [19, 20];

2. Interpolation of the samples at constant phase instead of at constant time, which results in the elimination of the effect of the increasing phase differences with time [21].

In both these approaches the interpolation of the samples can be performed using either a global or a piecewise polynomial approximation. For a robust interpolation an alternative Adaptive Stochastic Finite Elements formulation is proposed based on Newton-Cotes quadrature in simplex elements [18], which preserves monotonicity and extrema of the low number of samples. The resulting Unsteady Adaptive Stochastic Finite Elements (UASFE) approach [21] can be applied to problems in which the phase of the oscillatory samples is well-defined.

The paper is outlined as follows. The time-independent parametrization of the samples in combination with a global polynomial interpolation is developed in section 2 in application to an elastically mounted cylinder subject to a random uniform flow. The Adaptive Stochastic Finite Elements formulation is illustrated for a steady random transonic flow over a NACA0012 airfoil in section 3. In section 4 the combination of the interpolation of oscillatory samples at constant phase and the Adaptive Stochastic Finite Elements interpolation is applied to an elastically mounted airfoil with nonlinearity in the flow and the structure. The paper is concluded in section 5. The consistence of the approaches has been verified by comparison of results for analytical test problems with those of Monte Carlo simulations in previous studies [18, 19, 20, 21].

\section{Flow past an elastically mounted cylinder}

The concept of interpolating a time-independent parametrization of oscillatory samples instead of the time-dependent samples themselves is elaborated in section 2.1. In section 2.2 the fluid-structure interaction system of an elastically mounted cylinder is described. Results for a random free stream velocity are presented in section 2.3. 


\subsection{Interpolation of time-independent parametrization}

Consider a dynamical system subject to $n$ uncorrelated second-order random input parameters $\mathbf{a}(\omega)=\left\{a_{1}(\omega), \ldots, a_{n}(\omega)\right\}$, which governs an oscillatory response $u(\mathbf{x}, t, \omega)$

$$
\mathcal{L}(\mathbf{x}, t ; u(\mathbf{x}, t, \omega))=S(\mathbf{x}, t),
$$

with operator $\mathcal{L}$ and source term $S$ defined on domain $D \times T$, and appropriate initial and boundary conditions. The spatial and temporal dimensions are defined as $\mathbf{x} \in D$ and $t \in T$, respectively, with $D \subset \mathbb{R}^{d}, d=\{1,2,3\}$, and $T=\left[0, t_{\max }\right]$. A realization of the set of outcomes $\Omega$ of the probability space $(\Omega, \mathcal{F}, P)$ is denoted by $\omega \in \Omega$, with $\mathcal{F} \subset 2^{\Omega}$ the $\sigma$-algebra of events and $P$ a probability measure.

A suitable time-independent parametrization of the unknown oscillatory response surface $u(t, \omega)$ is given by the following representation $\tilde{u}(t, \omega)$ :

$$
\tilde{u}(t, \omega)=u_{0}(\omega)+\mathrm{e}^{\gamma(\omega)\left(t_{\max }-t\right)} A(\omega) u_{\text {period }}(\tau(t, \omega), \omega),
$$

with $\tau(t, \omega)=2 \pi\left(\phi(\omega)+\left(t-t_{\max }\right) f(\omega)\right)(\bmod 2 \pi)$. The argument $\mathbf{x}$ has been dropped here for convenience of the notation. The response $u(t, \omega)$ is parametrized by (2) in terms of the time-independent functionals: frequency $f(\omega)$; relative phase $\phi(\omega)$; amplitude $A(\omega)$; reference value $u_{0}(\omega)$; damping $\gamma(\omega)$; and normalized period shape $u_{\text {period }}(\tau(\omega), \omega)$, with $\tau(\omega) \in[0,2 \pi]$. These functions of $\omega$ are constructed using a Probabilistic Collocation approach [8] by solving (1) for the parameter values $\mathbf{a}_{k}$ which correspond to Gauss quadrature points in parameter space, with $k=1, \ldots, N_{\mathrm{s}}$ and $N_{\mathrm{s}}$ the number of samples. The realizations of the time-independent functionals $f_{k}, \phi_{k}, A_{k}, u_{0_{k}}, \gamma_{k}$, and $u_{\text {period, } k}(\tau)$ are then extracted from the computed samples $u_{k}(t)$. These realizations are finally interpolated using a global polynomial interpolation to the functions $f(\omega), \phi(\omega), A(\omega), u_{0}(\omega), \gamma(\omega)$, and $u_{\text {period }}(\tau, \omega)$, and substituted in representation (2). The mean and variance of approximation $\tilde{u}(t, \omega)$ are determined by numerically integrating of response surface (2). The distribution function is given by sorting the function $u-\omega$, with $\omega \in[0,1]$, to a monotonically increasing reconstruction.

Since the interpolation is performed at the level of the time-independent parameters, the interpolation accuracy is independent of time for a constant number of samples. This approach can be applied to the asymptotic region of oscillatory responses which allow for a time-independent parametrization of the form of (2).

\subsection{The elastically mounted cylinder system}

The two-dimensional fluid-structure interaction problem of an elastically-mounted circular cylinder in a laminar Navier-Stokes flow is given in Figure 1. The gas flow around the cylinder with diameter $d$ is governed by the two-dimensional compressible Navier-Stokes equations [22]. The cylinder is only free to move in the cross flow $y$ direction. The structural stiffness is modeled by a linear spring, which results in an angular natural frequency of the structure of $\omega_{\mathrm{n}}=\sqrt{0.1} \approx 0.316$. 


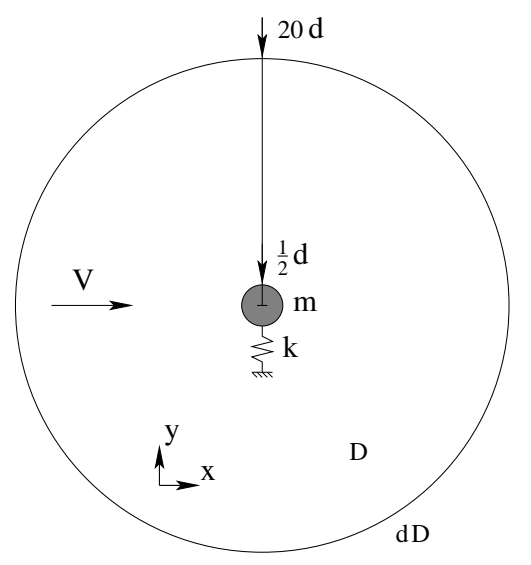

Figure 1: The elastically-mounted cylinder in a uniform free stream flow.

The flow equations are discretized on a circular spatial domain $D$ with diameter $40 d$ using a second-order finite volume method on a grid of $1.2 \cdot 10^{4}$ volumes. An Arbitrary Lagrangian-Eulerian formulation is employed to couple the fluid mesh with the movement of the structure. Time integration is performed using a BDF-2 method with a stepsize of $\Delta t=0.25$ until $t=250$. Initially the flow field is uniform and the cylinder is at rest with an initial deflection of $y_{\text {cyl }}=0.5 d$ with respect to its equilibrium position.

The undisturbed velocity in the $x$-direction, $V(\omega)$, is assumed to be uncertain described by a truncated lognormal distribution with a coefficient of variation of $C V_{\mathrm{V}}=10 \%$. The mean value of the velocity $\mu_{\mathrm{V}}=0.3$ corresponds to a Reynolds number of $R e=1000$. The truncated lognormal distribution limits the variation of the Reynolds number to the range for which the frequency $f$ of the periodic fluid motion is typically given by a Strouhal number of $S t=f d / V=0.2$. This corresponds for $\mu_{\mathrm{V}}$ to an angular frequency of $\omega_{\text {flow }}=0.38$. For this range the cylinder exhibits a period- 1 oscillation. The variation in $V(\omega)$ affects the frequency of the vortex shedding behind the cylinder and, therefore, influences the frequency of the motion of the cylinder.

\subsection{Efficient approximation of oscillatory responses}

The effect of the randomness in free stream velocity $V(\omega)$ on the evolution of the mean and the variance of the cylinder displacement $y(t, \omega)$ is shown in Figure 2. The approach based on the Probabilistic Collocation interpolation of the time-independent parametrization is in the figure referred to as Probabilistic Collocation for Limit Cycle Oscillations (PCLCO). The PCLCO approach is applied to the time domain in which the samples are in their asymptotic range. This corresponds to the asymptotic stochastic solution and part of the stochastic transient from the deterministic initial condition. Probabilistic Collocation applied directly to the time-dependent samples is used in the time domain in which the samples are in their transient. The locations where the methodology switches from Probabilistic Collocation to PCLCO are denoted by the 


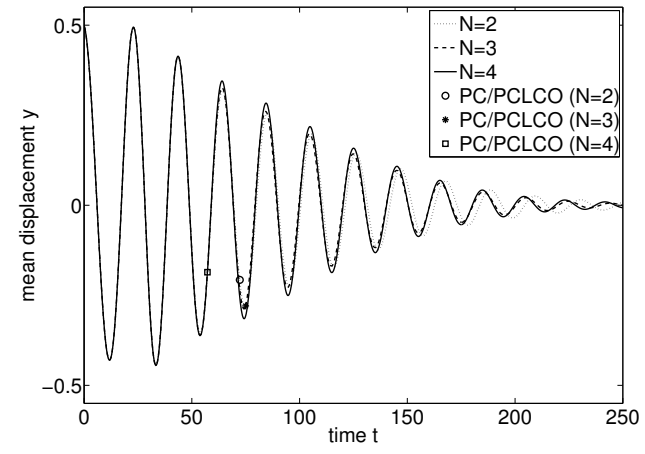

(a) mean

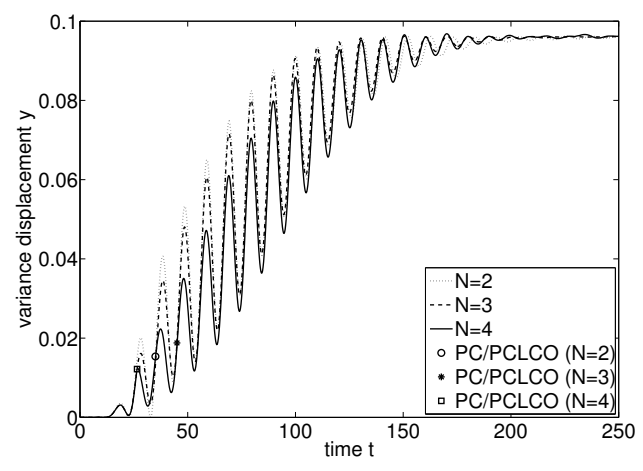

(b) variance

Figure 2: Deflection of the elastically-mounted cylinder for the combination of PCLCO and Probabilistic Collocation (PC).

symbols. Results for $N_{\mathrm{s}}=\{2,3,4\}$ samples are shown to demonstrate the level of convergence of the approximations.

Although the deterministic samples show a highly unsteady behavior, the stochastic behavior is asymptotically steady. The mean is a decaying oscillation to zero due the effect of the random free stream velocity $V(\omega)$ on the frequency of the oscillation. The frequency differences result in increasing phase differences in time, such that realizations with positive and negative sign increasingly cancel each other. The initially fast increasing variance demonstrates the importance of resolving the large effect of physical variations in unsteady problems. After the oscillatory stochastic transient the variance approaches a steady asymptotic value of approximately $9.6 \cdot 10^{-2}$ due to the asymptotically constant amplitude of the samples.

The coinciding Probabilistic Collocation approximations for $N_{\mathrm{s}}=\{2,3,4\}$ samples illustrate convergence for the short time interval $t<50$. For longer time integration Probabilistic Collocation would require a fast increasing number of samples with time to resolve the asymptotic stochastic behavior. However, the time-independent parametrization in PCLCO results in a converged approximation of the long-term stochastic behavior of the system for only $N_{\mathrm{s}}=4$ samples. These results illustrate that interpolation of the time-independent parametrization is an efficient approach for resolving the asymptotic effect of random parameters on oscillatory responses. In the stochastic transient $t \in[50,150]$ the results of PCLCO seem to converge less rapidly.

\section{Steady transonic flow over a NACA0012 airfoil}

Global polynomial interpolation of the samples gives accurate results for smooth responses as illustrated in the previous example. However, it can result in unreliable approximations near discontinuities. A more robust piecewise polynomial interpolation is, therefore, needed for problems with singularities. An alternative Adaptive 


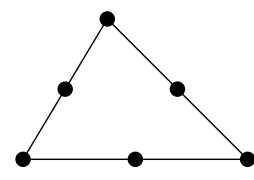

(a) Element

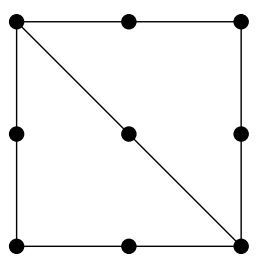

(b) Initial grid

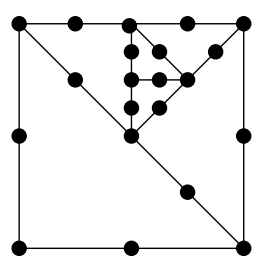

(c) Adapted grid

Figure 3: Discretization of two-dimensional parameter space using 2-simplex elements and second-degree Newton-Cotes quadrature points given by the dots.

Stochastic Finite Elements (ASFE) formulation based on Newton-Cotes quadrature in simplex elements is developed in section 3.1. The ASFE approach is applied to a steady transonic flow around a NACA0012 airfoil. The flow problem is described in section 3.2 and results are presented in section 3.3.

\subsection{Adaptive Stochastic Finite Elements with Newton-Cotes quadrature and simplex elements}

The non-intrusive Adaptive Stochastic Finite Elements (ASFE) interpolation based on Newton-Cotes quadrature points in simplex elements [18] employs a piecewise quadratic approximation of the response surface by dividing parameter space into $N_{\mathrm{e}}$ simplex elements. The quadratic approximation in the elements is constructed by performing deterministic solves for the values of the random parameters $\mathbf{a}(\omega)$ that correspond to the $\left(\begin{array}{c}n+2 \\ 2\end{array}\right)$ second-degree Newton-Cotes quadrature points in the elements shown in Figure 3a. The two-dimensional example of Figure 3 can geometrically be extended to higher-dimensional parameter spaces.

The initial discretization of parameter space by the adaptive scheme consists of the minimum of $N_{\mathrm{e}_{\text {ini }}}=n$ ! simplex elements and $N_{\mathrm{s}_{\text {ini }}}=3^{n}$ samples, see Figure $3 \mathrm{~b}$. The elements are adaptively refined as illustrated in Figure $3 c$ using a refinement measure based on the largest absolute eigenvalue of the Hessian, as measure of the curvature of the response surface approximation in the elements, weighted by the probability represented by the elements. The stochastic grid refinement is terminated when a $L_{\infty}$ convergence measure for the mean and standard deviation is reached or at a threshold for the maximum number of samples $\bar{N}_{\mathrm{s}}$.

Due to the location of the Newton-Cotes quadrature points the required number of deterministic solves is relatively low, since the deterministic samples are reused in successive refinements and the samples are used in approximating the response in multiple elements. Where necessary the elements are subdivided into $N_{\text {lin }}=2^{n}$ subelements with a linear approximation of the response without performing additional deterministic solves to preserve monotonicity and extrema of the samples. This prevents unphysical predictions due to overshoots and undershoots near singularities 


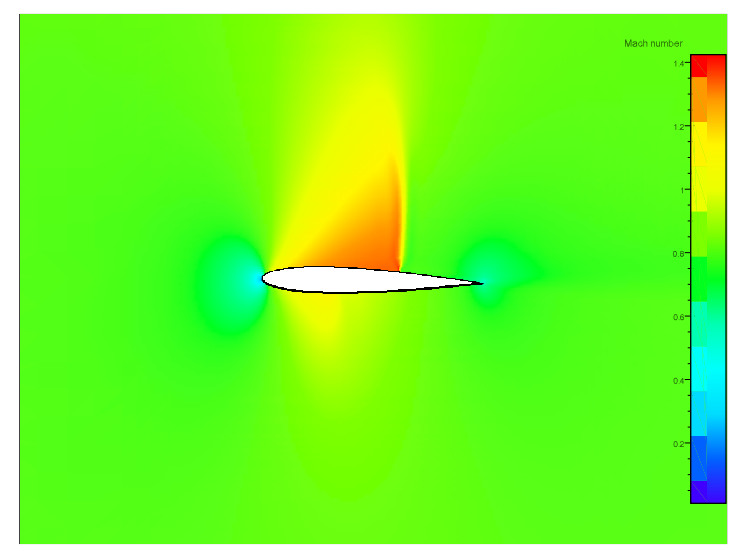

Figure 4: Transonic flow over a NACA0012 airfoil for the mean free stream Mach number $\mu_{\mathrm{Ma}_{\infty}}$.

at the expense that the method does not achieve exponential convergence for smooth responses as Gauss quadrature Stochastic Finite Elements methods can.

\subsection{The transonic airfoil flow problem}

The Adaptive Stochastic Finite Elements formulation is applied to a transonic Euler flow [23] over a NACA0012 airfoil subject to a random free stream Mach number $\mathrm{Ma}_{\infty}$. The distribution of $\mathrm{Ma}_{\infty}$ is a truncated lognormal with a mean Mach number of $\mu_{\mathrm{Ma} \infty}=0.8$ and a coefficient of variation of $C V_{\mathrm{Ma}_{\infty}}=1 \%$. The angle of attack is equal to $1.25^{\circ}$ and the airfoil has a chord with length $c$. The two-dimensional flow problem is discretized using a second-order upwind spatial finite volume scheme on a unstructured hexahedral mesh with $3 \cdot 10^{4}$ spatial volumes. The steady state solution is found by time integration with a CFL number of 0.5. In Figure 4 the flow field in terms of the local Mach number is shown for the mean value of the free stream Mach number $\mu_{\mathrm{Ma}_{\infty}}$. Above the wing a large supersonic domain can be identified for which Ma $>1$, which ends at a shock wave at $x \approx 0.6 c$. This shock wave appears as a discontinuity in probability space, which can result in a high sensitivity for small input variations.

\subsection{Robust approximation of discontinuities}

The effect of the random $\mathrm{Ma}_{\infty}$ is shown in Figure 5 in terms of the mean Mach number and the $99 \%$ confidence range along the upper surface of the airfoil. Adaptive Stochastic Finite Elements are applied with 4 elements, which results in 9 deterministic solves. The results are compared with Probabilistic Collocation based on 5 deterministic solves. Adaptive Stochastic Finite Elements predict that the randomness smears the shock wave in the mean Mach number along the upper surface around its deterministic location. The $99 \%$ confidence range shows that the nonlinear system 


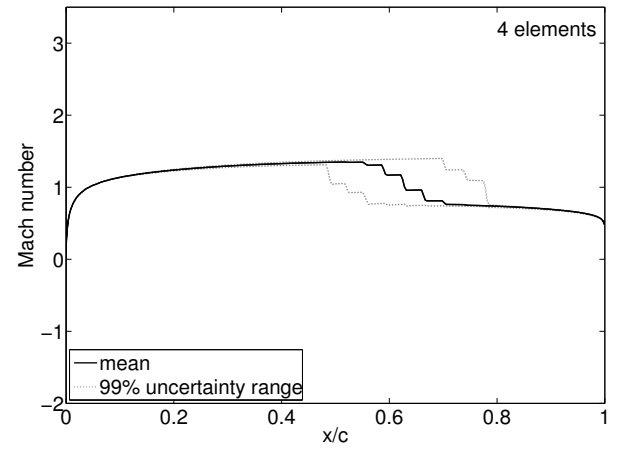

(a) ASFE, upper surface

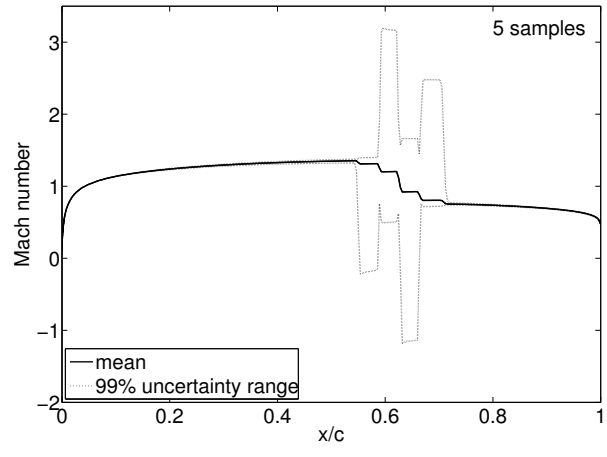

(b) PC, upper surface

Figure 5: Mean Mach number and 99\% uncertainty range of Adaptive Stochastic Finite Elements (ASFE) and Probabilistic Collocation (PC) along the upper surface of the transonic NACA0012 airfoil.

amplifies the $1 \%$ input randomness to a variation of the shock wave location between $x / c=0.5$ and $x / c=0.8$. The position of the shock wave is, therefore, sensitive to small variations in free stream Mach number, while the shock wave strength is nearly unaffected. The global polynomial interpolation of Probabilistic Collocation gives an oscillatory approximation near the discontinuity, which results in unphysical negative Mach numbers and unrealistically high Mach numbers of up to 3. Increasing the number of samples further increases the oscillatory behavior of the Probabilistic Collocation approximation. The robust extrema preserving approximation of the Adaptive Stochastic Finite Elements formulations, therefore, results for problems with discontinuous solutions in a more reliable approximation than a global polynomial interpolation.

\section{Stochastic bifurcation behavior of an elastically mounted airfoil}

The stochastic bifurcation behavior of an elastically mounted airfoil is studied using the second approach for unsteady problems based on interpolation of the oscillatory samples at constant phase. The interpolation is performed using the Adaptive Stochastic Finite Elements formulation described the previous section. In addition to a constant number of samples in time, interpolation at constant phase has the advantages over time-independent parametrization that it is not subject to parametrization error and that it is applicable to problems which do not allow for a time-independent parametrization. The concept of interpolation at constant phase is developed in in section 4.1. The deterministic system governing the elastically mounted airfoil with nonlinearity in the flow and the structure is described in section 4.2. Results of the straightforward implementation of the non-intrusive Unsteady Adaptive Stochastic Fi- 
nite Elements (UASFE) approach are presented in section 4.3.

\subsection{Interpolation at constant phase}

The non-intrusive approach is based on performing deterministic solves for the parameter values $\mathbf{a}_{k}$, which correspond to the Newton-Cotes quadrature points in the elements in parameter space. In order to interpolate the samples $u_{k}(t)$ at constant phase, first, their phase as function of time $\phi_{k}(t)$ is extracted from the deterministic solves $u_{k}(t)$. Second, the time series for the phase $\phi_{k}(t)$ are used to transform the samples $u_{k}(t)$ to functions of their phase $u_{k}^{*}\left(\phi_{k}\right)$ instead of time. Third, the transformed samples $u_{k}^{*}\left(\phi_{k}\right)$ are interpolated to the function $u^{*}(\phi, \omega)$ using Adaptive Stochastic Finite Elements interpolation. This step involves both the interpolation of the sampled phases $\phi_{k}(t)$ to the function $\phi(t, \omega)$ as well as the interpolation of the samples $u_{k}^{*}(\tilde{\phi})$ to the function $u^{*}(\tilde{\phi}, \omega)$ at constant phase $\tilde{\phi}$. Repeating the latter interpolation for all phases $\tilde{\phi}$ results in the function $u^{*}(\phi, \omega)$. Finally, transforming $u^{*}(\phi, \omega)$ back to $u(t, \omega)$ using $\phi(t, \omega)$ yields the unknown response surface of the system response as function of the random parameters $\mathbf{a}(\omega)$ and time $t$.

The phase $\phi_{k}(t)$ is extracted from the samples based on the local extrema of the time series $u_{k}(t)$. A trial and error procedure identifies a cycle of oscillation based on two or more successive local maxima. The selected cycle is accepted if the maximal error of its extrapolation in time with respect to the actual sample is smaller than a threshold value $\bar{\varepsilon}_{k}$ for at least one additional cycle length. The function for the phase $\phi_{k}(t)$ in the whole time domain $t \in T$ is constructed by identifying all successive cycles of $u_{k}(t)$ and extrapolation to $t=0$ and $t=t_{\max }$ before and after the first and last complete cycle, respectively. The phase is normalized to zero at the start of the first cycle and a user defined parameter determines whether the sample is assumed to attain a local maximum at $t=0$.

\subsection{The elastically mounted airfoil system}

The nonlinear structural stiffness is modeled by a cubic spring stiffness term in a twodegree-of-freedom model [24] for coupled pitch and plunge motion of the airfoil with chord $c$, see Figure $6 \mathrm{a}$. The values of the structural parameter correspond to those used in [25]. Randomness is introduced in the ratio of natural frequencies $\bar{\omega}(\omega)=\omega_{\xi} / \omega_{\alpha}$, with $\omega_{\xi}$ and $\omega_{\alpha}$ the natural frequencies of the airfoil in pitch and plunge, respectively. The randomness is described by a symmetric unimodal beta distribution with parameters $\beta_{1}=\beta_{2}=2$ around a mean of $\mu_{\bar{\omega}}=0.2$ in the domain $\bar{\omega}(\omega) \in[0.15 ; 0.25]$.

The aerodynamic force and moment coefficients are determined by solving the nonlinear Euler equations for inviscid flow [23] using a second-order finite volume scheme on an unstructured hexahedral mesh with $7.5 \cdot 10^{3}$ volumes in spatial domain $D$ with dimensions $30 c \times 20 c$. Time integration is performed using the BDF-2 method with stepsize $\Delta \tau=0.4$ until nondimensional time $\tau_{\max }=1000$. The initial condition 


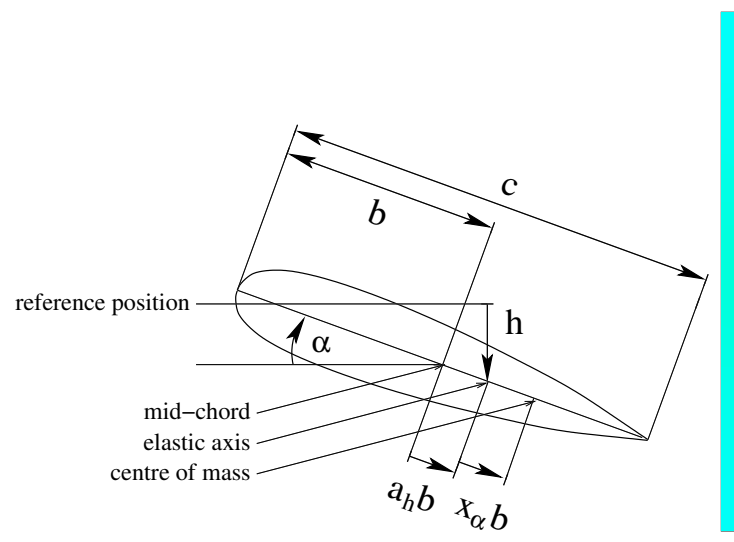

(a) structural model

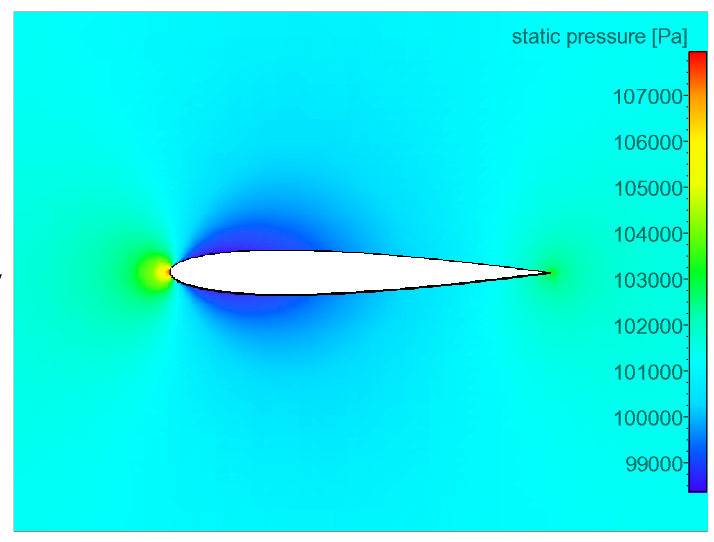

(b) initial flow conditions

Figure 6: The elastically mounted airfoil in uniform Euler flow.

is given by the steady state solution for the initial deflection of $\alpha(0)=0.1 \mathrm{deg}$ and $\xi(0)=0$ shown in Figure $6 \mathrm{~b}$ in terms of the static pressure field $p$. In the deterministic case the airfoil exhibits a supercritical Hopf-bifurcation at the linear flutter point $U^{*} / U_{L}^{*}=1$, which consists of a decaying oscillation below the flutter point and a diverging oscillation towards a limit cycle oscillation for $U^{*} / U_{L}^{*}>1$.

\subsection{Straightforward implementation of non-intrusive approach}

By propagating the randomness in the frequency ratio $\bar{\omega}(\omega)$ through the system for a range of values of bifurcation parameter $U^{*} / U_{L}^{*}$, the stochastic bifurcation behavior of the system can be explored. Due to the random frequency ratio $\bar{\omega}(\omega)$, the system bifurcation can no longer be described by a single deterministic flutter point, but rather by a P-bifurcation region for the probability distribution. A typical P-bifurcation can be recognized in the evolution of the probability density of amplitude $A_{\alpha}(\omega)$ for $U^{*} / U_{L}^{*} \in[0.95 ; 1.05]$ and $\Delta U^{*} / U_{L}^{*}=0.0125$ at $\tau=800$ in Figure 7. In the prebifurcation domain of $U^{*} / U_{L}^{*} \leq 0.9625$ the probability density resembles a Dirac delta function at $A_{\alpha}=0$. At $U^{*} / U_{L}^{*}=0.9875$ the probability still has a maximum at $A_{\alpha}=0$ and decays monotonically to zero for positive values of $A_{\alpha}$. The randomness in $\bar{\omega}(\omega)$, therefore, results in an earlier onset of unstable post-flutter behavior than a deterministic analysis would point out. At the deterministic bifurcation point $U^{*} / U_{L}^{*}=1$ the probability density develops a local maximum at a positive amplitude $A_{\alpha}$ of $5.7 \mathrm{deg}$. The local maximum increases and occurs at increasing values of $A_{\alpha}$ until it turns into the global most probable point at $U^{*} / U_{L}^{*}=1.025$. In the post-bifurcation domain of $U^{*} / U_{L}^{*} \geq 1.025$ the unimodal probability density function allows for positive $A_{\alpha}$ values only.

Due to the straightforward implementation of the non-intrusive Unsteady Adaptive Stochastic Finite Elements formulation these results are obtained by reusing a deterministic solver for fluid-structure interaction simulation. The increased insight in the 


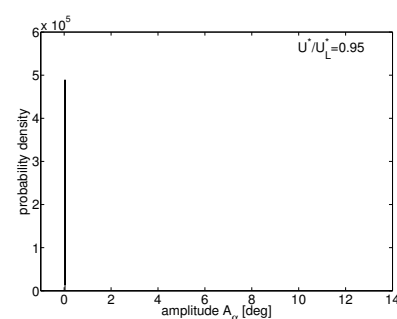

(a) $U^{*} / U_{L}^{*}=0.95$

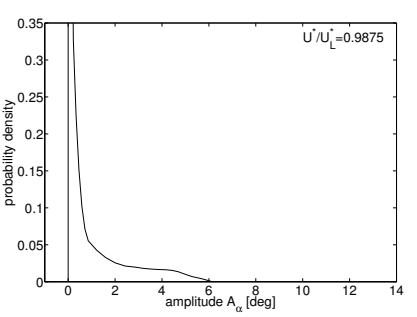

(d) $U^{*} / U_{L}^{*}=0.9875$

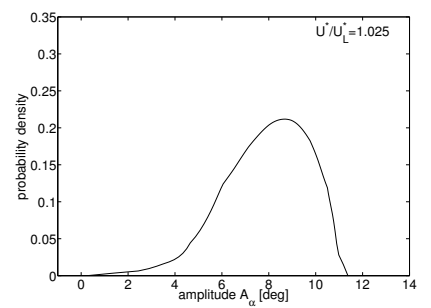

(g) $U^{*} / U_{L}^{*}=1.025$

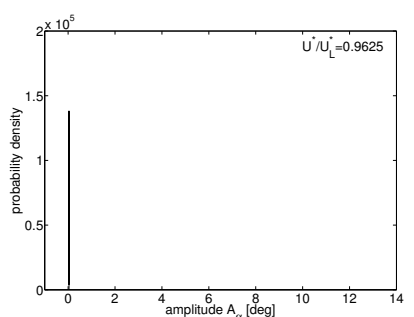

(b) $U^{*} / U_{L}^{*}=0.9625$

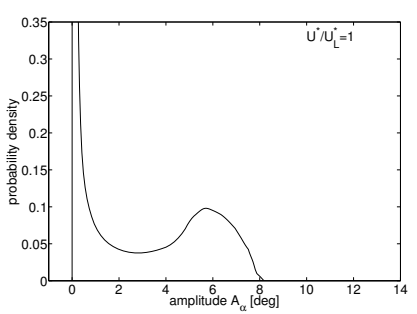

(e) $U^{*} / U_{L}^{*}=1$

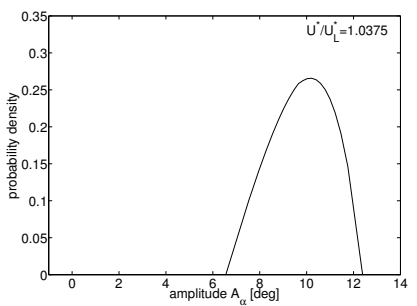

(h) $U^{*} / U_{L}^{*}=1.0375$

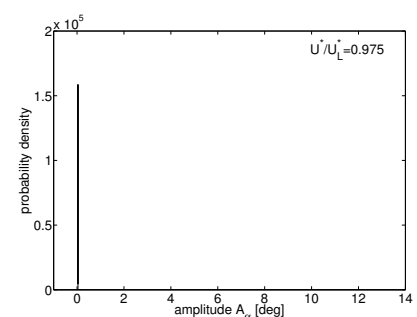

(c) $U^{*} / U_{L}^{*}=0.975$

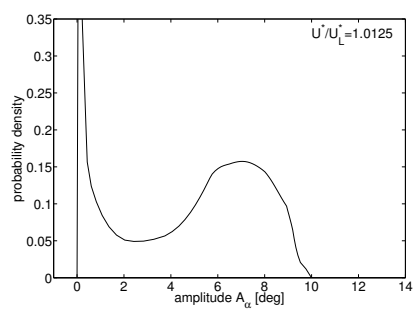

(f) $U^{*} / U_{L}^{*}=1.0125$

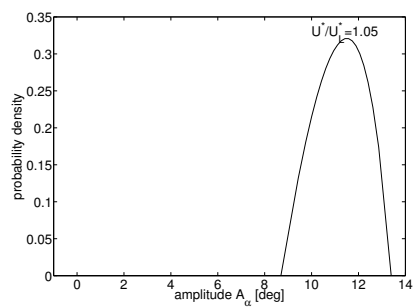

(i) $U^{*} / U_{L}^{*}=1.05$

Figure 7: Probability density of amplitude $A_{\alpha}(\omega)$ for $U^{*} / U_{L}^{*} \in[0.95 ; 1.05]$ at $\tau=800$ for the elastically mounted airfoil. 
bifurcation behavior of the elastically mounted airfoil through these detailed stochastic results demonstrates the additional value of a stochastic analysis compared to a deterministic simulation for complex physical systems.

\section{Conclusions}

Modeling physical variability is vital for making reliable flow and fluid-structure interaction predictions, since unsteadiness and shock waves can amplify input randomness to the onset of unstable behavior. In this paper, two methodologies for unsteady problems with discontinuities are developed based on interpolation of a time-independent parametrization of the samples and interpolation of the samples at constant phase. These two ideas result both in a constant accuracy in time with a constant number of samples, in contrast with the usually fast increasing number of samples for other methods. Interpolation at constant phase has the advantages over time-independent parametrization that it is not subject to parametrization error and that it is applicable to problems which do not allow for a time-independent parametrization.

The interpolation of the samples is performed using both a global and a piecewise polynomial approximation. For a robust interpolation an alternative Adaptive Stochastic Finite Elements (ASFE) formulation is developed based on Newton-Cotes quadrature in simplex elements, which preserves monotonicity and extrema of the low number of samples. The resulting Unsteady Adaptive Stochastic Finite Elements (UASFE) formulation can be applied to problems in which the phase of the oscillatory samples is well-defined.

The converged approximation of the asymptotic stochastic behavior of an elastically mounted cylinder in random uniform flow based on four deterministic samples shows the efficiency of the time-independent parametrization approach. The steady random transonic Mach number flow around a NACA0012 airfoil shows that the Adaptive Stochastic Finite Elements formulation results in a robust approximation of discontinuous response surfaces. The application of the Unsteady Adaptive Stochastic Finite Elements approach to study the stochastic bifurcation behavior of an elastically mounted airfoil illustrates that the implementation of the non-intrusive approach is straightforward by reusing an existing deterministic solver.

\section{Acknowledgments}

This research was supported by the Technology Foundation STW, applied science division of NWO and the technology programme of the Ministry of Economic Affairs. 


\section{References}

[1] J.M. Hammersley, D.C. Handscomb, "Monte Carlo methods", Methuen's monographs on applied probability and statistics, Methuen, London, 1964.

[2] I.M. Babuška, R. Tempone, G.E. Zouraris, "Galerkin finite element approximations of stochastic elliptic partial differential equations", SIAM J. Numer. Anal., 42, 800-825, 2004.

[3] R.G. Ghanem, P.D. Spanos, "Stochastic finite elements: a spectral approach", Springer-Verlag, New York, 1991.

[4] S. Hosder, R. W. Walters, R. Perez, "A non-intrusive polynomial chaos method for uncertainty propagation in CFD simulations", 44 4 th AIAA Aerospace Sciences Meeting and Exhibit, Reno, NV, AIAA-2006-891, 2006.

[5] M.T. Reagan, H.N. Najm, R.G. Ghanem, O.M. Knio, "Uncertainty quantification in reacting-flow simulations through non-intrusive spectral projection", Combust. Flame, 132, 545-555, 2003.

[6] J.A.S. Witteveen, H. Bijl, "A monomial chaos approach for efficient uncertainty quantification in nonlinear problems", SIAM J. Sci. Comput., 30, 1296-1317, 2008.

[7] D.B. Xiu, G.E. Karniadakis, “The Wiener-Askey polynomial chaos for stochastic differential equations", SIAM J. Sci. Comput., 24, 619-644, 2002.

[8] I.M. Babuška, F. Nobile, R. Tempone, "A stochastic collocation method for elliptic partial differential equations with random input data", SIAM J. Numer. Anal., 45, 1005-1034, 2007.

[9] L. Mathelin, M. Y. Hussaini, Th.A. Zang, "Stochastic approaches to uncertainty quantification in CFD simulations", Num. Alg., 38, 209-236, 2005.

[10] M.A. Tatang, "Direct incorporation of uncertainty in chemical and environmental engineering systems", PhD thesis, MIT, Cambridge, 1995.

[11] P.S. Beran, C.L. Pettit, D.R. Millman, "Uncertainty quantification of limit cycle oscillations", J. Comput. Phys., 217, 217-247, 2006.

[12] C.L. Pettit, P.S. Beran, "Spectral and multiresolution Wiener expansions of oscillatory stochastic processes", J. Sound Vib., 294, 752-779, 2006.

[13] D.R.Millman, P.I. King, P.S. Beran, "Airfoil pitch-and-plunge bifurcation behavior with Fourier chaos expansions", J. Aircraft, 42, 376-384, 2005.

[14] M.K. Deb, I.M. Babuška, J.T. Oden, "Solution of stochastic partial differential equations using Galerkin finite element techniques", Comput. Methods Appl. Mech. Eng., 190, 6359-6372, 2001.

[15] O.P. Le Maître, O.M. Knio, H.N. Najm, R.G. Ghanem, "Uncertainty propagation using Wiener-Haar expansions", J. Comput. Phys., 197, 28-57, 2004.

[16] O.P. Le Maître, H.N. Najm, R.G. Ghanem, O.M. Knio, "Multi-resolution analysis of Wiener-type uncertainty propagation schemes", J. Comput. Phys., 197, 502-531, 2004.

[17] X.L. Wan, G.E. Karniadakis, "An adaptive multi-element generalized polynomial chaos method for stochastic differential equations", J. Comput. Phys., 209, 617-642, 2005. 
[18] J.A.S. Witteveen, G.J.A. Loeven, H. Bijl, "An adaptive stochastic finite elements approach based on Newton-Cotes quadrature in simplex elements", submitted, 2007.

[19] J.A.S. Witteveen, G.J.A. Loeven, S. Sarkar, H. Bijl, "Probabilistic Collocation for period-1 limit cycle oscillations", J. Sound Vib., 311, 421-439, 2008.

[20] J.A.S. Witteveen, H. Bijl, "An unsteady adaptive stochastic finite elements formulation for rigid-body fluid-structure interaction", submitted, 2007.

[21] J.A.S. Witteveen, H. Bijl, "An alternative unsteady adaptive stochastic finite elements formulation based on interpolation at constant phase", submitted, 2008.

[22] J.D. Anderson, "Fundamentals of aerodynamics", McGraw-Hill, New York, 1991.

[23] A.J. Chorin, J.E. Marsden, "A mathematical introduction to fluid mechanics", Springer-Verlag, New York, 1979.

[24] Y. Fung, "An introduction to aeroelasticity", Dover Publications, New York, 1969.

[25] B.H.K. Lee, L.Y. Jiang, Y.S. Wong, "Flutter of an airfoil with a cubic nonlinear restoring force", $39^{\text {th }}$ AIAA/ASME/ASCE/AHS/ASC Structures, Structural Dynamics, and Materials Conference, Long Beach, CA, AIAA-1998-1725, 1998. 